\title{
Some Structures of Hemirings
}

\author{
Md. Yasin Ali ${ }^{1,3}$, Kanak Ray Chowdhury ${ }^{2}$, Abeda Sultana ${ }^{3}$, Nirmal Kanti Mitra ${ }^{4}$ \\ ${ }^{1}$ School of Science and Engineering, University of Information Technology \& Sciences, Dhaka, Bangladesh \\ ${ }^{2}$ Department of Mathematics, Mohammadpur Model School and College, Mohammadpur, Dhaka, Bangladesh \\ ${ }^{3}$ Department of Mathematics, Jahangirnagar University, Savar, Bangladesh \\ ${ }^{4}$ Mathematical and Physical Sciences, Bangladesh University of Business and Technology, Dhaka, Bangladesh
}

Email address:

ali.mdyasin56@gmail.com (Md. Y. Ali)

\section{To cite this article:}

Md. Yasin Ali, Kanak Ray Chowdhury, Abeda Sultana, Nirmal Kanti Mitra. Some Structures of Hemirings. Pure and Applied Mathematics Journal. Vol. 6, No. 1, 2017, pp. 45-50. doi: 10.11648/j.pamj.20170601.16

Received: January 27, 2017; Accepted: February 8, 2017; Published: March 1, 2017

\begin{abstract}
Hemirings appear in a natural manner, in some applications to the theory of automata, the theory of formal languages, graph theory, design theory and combinatorial geometry. Recently, the notions of hemirings with special structures were introduced. But still now there are no complete structural properties of hemirings. In this paper we try to investigate some structures of hemirings. This is done by introducing some examples of hemirings.
\end{abstract}

Keywords: Hemirings, Zerosumfree Hemirings, Simple Hemirings

\section{Introduction}

Hemirings which are regarded as a generalization of rings have been found useful in solving problems in different areas of applied mathematics and computer sciences $[2,3,5]$. So many researchers have studied different aspects of hemirings. J. S. Golan [1] quoted some special classes of hemirings such as idempotent hemiring, zerosumfree hemiring, regular hemiring and so on. M. Gulistan et al. [7] characterize the gamma hemirings through level cuts in terms of generalized fuzzy (left, right, prime, semiprime) gamma ideals of gamma hemirings. Zhan et al. [4] gave the concept of $h$ -hemiregularity of hemirings and investigated some properties in terms of fuzzy theory. Yin et al. [10] gave the notions of $h$-semisimple hemirings and invested the characterizations by fuzzy ideals. Also some researches on hemirings are focused on fuzzy ideals $[6,12,14-16]$, soft set theory [11] and bipolar fuzzy theory [17]. In this paper we characterized some classes of hemirings especially zerosumfree hemiring, idempotent hemiring, regular hemiring and simple hemiring also counter examples are given. This paper is organized as follows: in section 2 we describe some preliminaries on hemirings, in section 3 some structures on hemirings are given, in section 4 the structures of simple hemirings are described.

\section{Preliminaries}

Definition 2.1 [12]: Let $R$ be non empty set with binary operation $*$. Then the algebraic structure $(R ; *)$ is called a semigroup if $\forall a, b, c \in R ; a *(b * c)=(a * b) * c$.

Definition 2.2 [1]: A hemiring is a nonempty set $R$ on which operations of addition and multiplication have been defined such that the following conditions are satisfied:

$\left[H_{1}\right]:(R ;+)$ is a commutative monoid with identity element 0 ;

$\left[\mathrm{H}_{2}\right]:(\mathrm{R} ;)$ is a semigroup;

$\left[\mathrm{H}_{3}\right]$ : Multiplication distributes over addition from either side;

$\left[\mathrm{H}_{4}\right]$ : The element 0 is the absorbing element of the multiplication i.e., $0 . r=0=r .0$.

Example 2.2 (a): The set $2 Z_{0}^{+}$is an infinite hemiring with usual addition and multiplication.

Example $2.2(b):(R=\{0, a, b\} ;+, \cdot)$ is a finite hemiring, where addition operation $(+)$ and multiplication operation $(\cdot)$ are as follows: 


\begin{tabular}{c|ccc}
+ & 0 & $\mathrm{a}$ & $\mathrm{b}$ \\
\hline 0 & 0 & $\mathrm{a}$ & $\mathrm{b}$ \\
$\mathrm{a}$ & $\mathrm{a}$ & $\mathrm{a}$ & $\mathrm{b}$ \\
$\mathrm{b}$ & $\mathrm{b}$ & $\mathrm{b}$ & $\mathrm{b}$
\end{tabular}

\begin{tabular}{c|ccc}
. & 0 & $\mathrm{a}$ & $\mathrm{b}$ \\
\hline 0 & 0 & 0 & 0 \\
$\mathrm{a}$ & 0 & $\mathrm{a}$ & 0 \\
$\mathrm{~b}$ & 0 & 0 & $\mathrm{a}$
\end{tabular}

Example 2.2 (c): The set $S=\{0,1,2,3\}$ with the following Cayley Tables:

\begin{tabular}{l|llll}
+ & 0 & 1 & 2 & 3 \\
\hline 0 & 0 & 1 & 2 & 3 \\
1 & 1 & 1 & 2 & 3 \\
2 & 2 & 2 & 2 & 3 \\
3 & 3 & 3 & 3 & 2
\end{tabular}

\begin{tabular}{c|llll}
. & 0 & 1 & 2 & 3 \\
\hline 0 & 0 & 0 & 0 & 0 \\
1 & 0 & 1 & 1 & 1 \\
2 & 0 & 1 & 1 & 1 \\
3 & 0 & 1 & 1 & 1
\end{tabular}

is a finite hemiring

Definition 2.3: If $R$ is a hemiring then an element $1 \in R$ (if 1 exists) and $1 \neq 0$ is called an identity element of $R$ if $1 \cdot a=a=a \cdot 1 ; \forall a \in R$. And if $R$ contains an identity element then it is called a hemiring with identity.

Example 2.3 (a): The set of natural numbers with zero is a hemiring with identity.

Definition 2.4: If $R$ is a hemiring, then a non-empty $A \subseteq R$ is called a sub-hemiring of $R$ if it contains zero and is closed with respect to the addition and multiplication of $R$.

Example 2.4 (a): If $R$ is a hemiring, then

$P(R)=\{0\} \cup\{r+1: r \in R\}$ is a sub-hemiring of $R$.

Definition 2.5: A hemiring $R$ is said to be commutative if

$(R ;)$ is commutative i.e.,

$$
a \cdot b=b \cdot a ; \forall a, b \in R \text {. }
$$

Example 2.5 (a): Consider the set $R=\{0, a, 1\}$ with the following two operations:

\begin{tabular}{l|lll}
+ & 0 & $\mathrm{a}$ & 1 \\
\hline 0 & 0 & $\mathrm{a}$ & 1 \\
$\mathrm{a}$ & $\mathrm{a}$ & $\mathrm{a}$ & $\mathrm{a}$ \\
1 & 1 & $\mathrm{a}$ & 1
\end{tabular}

\begin{tabular}{l|lll}
$\cdot$ & 0 & $\mathrm{a}$ & 1 \\
\hline 0 & 0 & 0 & 0 \\
$\mathrm{a}$ & 0 & $\mathrm{a}$ & $\mathrm{a}$ \\
1 & 0 & $\mathrm{a}$ & 1
\end{tabular}

Then $(R ;+, \cdot)$ is a commutative hemiring

Proposition 2.6: A set $R$ containing two distinct elements 0 and 1 and where operations + and $\cdot$ are defined, is a commutative hemiring iff the following four conditions are satisfied:

$\forall a, b, c, d, e \in R$;

(1) $a+0=0+a$

(2) $a \cdot 1=a$

(3) $0 . a=0$

(4) $[(a \cdot e+b)+c] \cdot d=d \cdot b+[a \cdot(e \cdot d)+c \cdot d]$

Proof: Surely any commutative hemiring satisfied conditions (1)-(4). Conversely, assume that these four conditions are satisfied. If

$$
\begin{aligned}
b, d \in R \Rightarrow b \cdot d & =[(0 \cdot 0+b)+0] \cdot d \\
& =d \cdot b[0 \cdot(0 \cdot d)+0 \cdot d] \\
& =d \cdot b
\end{aligned}
$$

So multiplication is commutative. If

$$
\begin{aligned}
a, b \in R \Rightarrow a+b & =[(a \cdot 1+b)+0] \cdot 1 \\
& =1 \cdot b+[a \cdot(1 \cdot 1)+0 \cdot 1] \\
& =b+a
\end{aligned}
$$

And so additive is commutative. If

$$
\begin{aligned}
a, e, d \in R \Rightarrow(a \cdot e) \cdot d & =[(a \cdot e+0)+0] \cdot d \\
& =d \cdot 0+[a \cdot(e \cdot d)+0 \cdot d] \\
& =0+[a \cdot(e \cdot d)+0] \\
& =a \cdot(e \cdot d)
\end{aligned}
$$

Multiplication is associative. If

$$
\begin{aligned}
a, b, c \in R \Rightarrow(a+b)+c & =(b+a)+c \\
& =[(b+a)+c] \cdot 1 \\
& =1 \cdot a+[b \cdot(1 \cdot 1)+c \cdot 1] \\
& =a+(b+c)
\end{aligned}
$$

And so addition is associative. Finally if

$$
\begin{aligned}
a, b, d \in R \Rightarrow(a+b)+d & =[(a \cdot 1+b)+0] \cdot d \\
& =d \cdot b+[a \cdot(1 \cdot d)+c \cdot d] \\
& =d \cdot b+a \cdot d \\
& =a \cdot d+b \cdot d
\end{aligned}
$$

And so multiplication distribute over addition. Thus $R$ is a commutative hemiring.

Definition 2.7 [1]: An element $r$ of a hemiring $R$ is additively idempotent if $r+r=r ; \forall r \in R$. The set

$I^{+}(R)$ of all additively idempotent elements of $R$ is non-empty since it contains 0 . The hemiring $R$ is additively idempotent if $I^{+}(R)=R$.

Example $2.7(a)$ : The fuzzy algebra $(F=[0,1] ; \vee, T)$; where $\vee=\max$ and $T$ is t-norm is an additive idempotent hemiring.

Definition 2.8 [1]: An element $a$ of a hemiring $R$ is multiplicatively idempotent if $a^{2}=a ; \forall a \in R$. The set $I^{+}(R)$ of all multiplicatively idempotent elements of $R$ is non-empty since it contains 0 . The hemiring $R$ is multiplicatively idempotent if $I^{\times}(R)=R$.

Example 2.8 (a): The hemiring $R=\{0, a, 1\}$ with the following two operations:

\begin{tabular}{c|ccc}
+ & 0 & $\mathrm{a}$ & 1 \\
\hline 0 & 0 & $\mathrm{a}$ & 1 \\
$\mathrm{a}$ & $\mathrm{a}$ & $\mathrm{a}$ & $\mathrm{a}$ \\
1 & 1 & $\mathrm{a}$ & 1
\end{tabular}

\begin{tabular}{l|lll}
. & 0 & $\mathrm{a}$ & 1 \\
\hline 0 & 0 & 0 & 0 \\
$\mathrm{a}$ & 0 & $\mathrm{a}$ & $\mathrm{a}$ \\
1 & 0 & $\mathrm{a}$ & 1
\end{tabular}

is multiplicatively idempotent.

Definition 2.9 [1]: The hemiring $R$ is idempotent if it is both additively and multiplicatively idempotent i.e.,

$I(R)=I^{+}(R) \cap I^{\times}(R)$. 
Example 2.9 (a): The hemiring $R=\{0, a\}$ and addition and multiplication defined as maximum and minimum respectively is an idempotent hemiring.

Example 2.9 (b): Let $R$ be set. The set of subsets of $R$, $P(S)$ or the set of finite subsets of $P^{F i n}(S)$ where addition and multiplication define as union and intersection respectively are both idempotent hemiring. Here $0=\phi$.

Definition 2.10 [18]: A semigroup $(R ;+)$ satisfying the identity $a=a+b+a$ is a rectangular band.

Definition 2.11 [18]: A semigroup $(R ;$; ) satisfying the identity $a=a b a$ is a rectangular band.

Definition 2.12 [1]: An element $a$ of a hemiring $R$ is multiplicatively sub-idempotent if $a+a^{2}=a$, and a hemiring $R$ is multiplicatively sub-idempotent if each of its elements is multiplicatively sub-idempotent.

Example 2.12 (a): The hemiring

$(R=\{0, a\} ;,+, \cdot)$ is multiplicatively sub-idempotent where addition operation $(+)$ and multiplication operation $(\cdot)$ are defined as follows:

\begin{tabular}{c|cc}
+ & 0 & $\mathrm{a}$ \\
\hline 0 & 0 & $\mathrm{a}$ \\
$\mathrm{a}$ & $\mathrm{a}$ & $\mathrm{a}$
\end{tabular}

\begin{tabular}{l|ll}
. & 0 & $\mathrm{a}$ \\
\hline 0 & 0 & 0 \\
$\mathrm{a}$ & 0 & $\mathrm{a}$
\end{tabular}

Definition 2.13 [18]: An element $a$ of a hemiring $R$ is almost idempotent if $a+a^{2}=a^{2}$, and a hemiring $R$ is almost idempotent if each of its elements is almost idempotent.

Example 2.13 (a): The hemiring $(R=\{0, b\} ;,+, \cdot)$ is almost idempotent where addition operation $(+)$ and multiplication operation $(\cdot)$ are defined as follows:

\begin{tabular}{c|cc}
+ & 0 & $\mathrm{~b}$ \\
\hline 0 & 0 & $\mathrm{~b}$ \\
$\mathrm{~b}$ & $\mathrm{~b}$ & $\mathrm{~b}$
\end{tabular}

\begin{tabular}{c|cc}
$\cdot$ & 0 & $\mathrm{~b}$ \\
\hline 0 & 0 & 0 \\
$\mathrm{~b}$ & 0 & $\mathrm{~b}$
\end{tabular}

Definition 2.14: A hemiring $(R ;+, \cdot)$ is said to be zerosquare hemiring if $\exists x \in R$ such that $x^{2}=0$.

\section{Some Structures of Hemirings}

In this section, we discuss about zerosumfree hemiring, idempotent hemiring and hemiregular hemiring.

Definition 3.1: A hemiring $(R ;+, \cdot)$ is said to be zerosumfree hemiring if $\exists x \in R$ such that $x+x=0$. implies that $x=0$.

Example $3.1(a)$ : The hemiring

$(R=\{0, a, b\} ;+\cdot)$, where addition operation $(+)$ and multiplication operation $(\cdot)$ defined as follows:
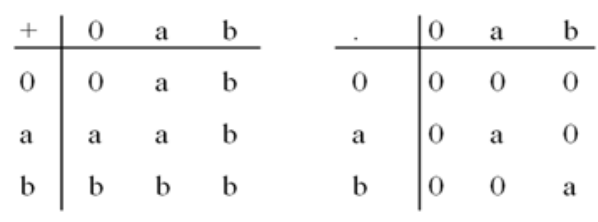

is a zerosumfree hemiring.

Example 3.1 (b): The set $N_{0}$ of non-negative integers with usual addition and multiplication of integers is a zerosumfree hemiring.

Proposition 3.2: An additively idempotent hemiring $R$ is zerosumfree iff $\exists r, r^{\prime} \in R$ such that $r+r^{\prime}=0$ implies that $r=r^{\prime}=0$.

Proof: If $r+r^{\prime}=0$ then

$r=r+0=r+\left(r+r^{\prime}\right)=(r+r)+r^{\prime}=r+r^{\prime}=0 \quad$ and similarly, $r^{\prime}=0$.

Conversely if, $r=r^{\prime}=0$, then, $r+r^{\prime}=0$.

Proposition 3.3: A hemiring $R$ with unity is a zerosumfree if $\exists t \in R$ satisfying $t=t+1$, if $r+r^{\prime}=0$ implies that $r=r^{\prime}=0 ;$ for $r, r^{\prime} \in R$.

$$
\text { Proof: } \begin{aligned}
0 & =\left(r+r^{\prime}\right) t=r t+r^{\prime} t \\
& =r(1+t)+r^{\prime}(1+t) \\
& =r(1+t)+r^{\prime}(1+1+t) \\
& =r(1+t)+r^{\prime}(1+1+t) \\
& =\left(r+r^{\prime}\right)+r^{\prime}+\left(r+r^{\prime}\right) t \\
& =r^{\prime}
\end{aligned}
$$

and so, $r=r+r^{\prime}=0$ as well.

Proposition 3.4: If $(R ;+, \cdot)$ is a zerosumfree hemiring. Then $a+a b=a ; \forall a, b \in R$ iff $a b=0$.

Proof: Consider $a+a b=a ; \forall a, b \in R$;

$\Rightarrow a+a+a b=a+a$

$\Rightarrow a b=0 . \quad[\because S$ is zerosumfree hemiring, so $a+a=o$ ]

Conversely, suppose $a b=0 ; \forall a, b \in R$

$\Rightarrow a+a b=a+0$

$\Rightarrow a+a b=a$.

Theorem 3.5: Suppose $R$ is zerosumfree hemiring. Then $R$ is multiplicatively sub-idempotent hemiring iff $R$ is zerosquare hemiring.

Proof: By definition of zerosumfree hemiring $a+a=o$; $\forall a \in R$.

Also we have $a+a^{2}=a ; \forall a \in R$.

$\Rightarrow a+a+a^{2}=a+a$.

$\Rightarrow a^{2}=0 \quad[\because a+a=0]$

Thus $R$ is zerosquare hemiring.

To prove the converse part let us take $a^{2}=0$

$\Rightarrow a+a^{2}=a+0$.

$\Rightarrow a+a^{2}=a ; \forall a \in R$.

Therefore $R$ is multiplicatively sub-idempotent hemiring. $a+a b=a ; \forall a, b \in R$.

Lemma 3.6: If $a, b$ are two idempotent elements of a 
hemiring $R$, then $a b$ is also idempotent.

Proof: Since $a, b$ are two idempotent elements of a hemiring, then we have

$a^{2}=a$ and $b^{2}=b$.

Now, $\begin{aligned}(a b)^{2} & =a^{2} b^{2} \\ & =a b\end{aligned}$

Hence $a b$ is an idempotent of $R$.

Proposition 3.7: If $a, b, c$ and are elements of an additively idempotent hemiring $R$ satisfying $a+c=b$ and $b+d=a$, then $a=b$.

Proof: If is additively idempotent hemiring. i.e, $a+a=0$.

$$
\text { Now, } \begin{aligned}
a & =a+b+d & & {[\because a=b+d] } \\
& =a+a+c+d & & {[\because b=a+c] } \\
& =a+c+d & & {[\because a+a=a] } \\
& =b+d+c+d & & \\
& =b+d+d+c & & \\
& =b+d+c & & \\
& =a+c & & \\
& =b & & {[\because b=a+c] }
\end{aligned}
$$

Proposition 3.8: Let $R$ be a multiplicatively idempotent hemiring satisfying the condition that $a+a b+a=a=a+b a+a ; \forall a, b \in R$.

Then $R$ is additively idempotent and in addition, satisfies the condition that

$$
a b+b a=a b ; \forall a, b \in R .
$$

Proof: If $a \in R$ then,

$$
\begin{aligned}
a+a & =(a+a)^{2}=a+a+a+a \\
& =a+a^{2}+a^{2}+a \\
& =a+a(a+a)+a \\
& =a
\end{aligned}
$$

And so $R$ is additively idempotent. Moreover, if $a, b \in R$ then

$$
\begin{aligned}
a b+b a & =a b+b a+a b[\text { by lemma }] \\
& =(a b+b a+a b)^{2} \\
& =a b(a b+b a+a b)+b a(a b+b a+a b) \\
& +a b(a b+b a+a b) \\
& =[a b(a b) a+a b)]+[b a b+(b a b) a+b a b) \\
& +[a b+(a b) a+a b] \\
& =a b+b(a b)+a b \\
& =a b
\end{aligned}
$$

Theorem 3.9: Let $R$ be a hemiring with multiplicatively identity 1 satisfying the identity $a+b=1 ; \forall a, b \in R$.

If $(R ;+)$ is rectangular band then $R$ is almost idempotent hemiring.

Proof: $a+b=1$

$\Rightarrow a^{2}+a b=a$

$\Rightarrow a^{2}+a b+a^{2}=a+a^{2}$

$\Rightarrow a(a+b+a)=a+a^{2}$

$\Rightarrow a^{2}=a+a^{2}$

$$
\begin{aligned}
& \text { Again, } a+b=1 \\
& \Rightarrow a b+b^{2}=b \\
& \Rightarrow b^{2}+a b+b^{2}=b^{2}+b \\
& \Rightarrow(b+a+b) b=b^{2}+b \\
& \Rightarrow b^{2}=b^{2}+b
\end{aligned}
$$

Hence $R$ is almost idempotent hemiring.

Proposition 3.10: If $(R ;)$ is a rectangular band, then $a b$ is multiplicatively idempotent $\forall a, b \in R$.

Proof: Since $(R ;)$ is a rectangular band, so there exists an element $b$ in $R$ such that

$a b a=a$.

Now, $(a b)^{2}=a b a b=(a b a) b=a b$.

Hence $a b$ is multiplicatively idempotent.

Definition 3.11: A hemiring $R$ is said to be $h$-hemiregular hemiring if for each $a \in S$, there exists $x, y, z \in R$ such that

$a+a x a+z=a y a+z$.

Example 3.11 (a): Let $R$ be the set of all non-negative integers with an element $\infty$ such that $\infty \geq x ; \forall x \in N_{0}$.

Consider two operations: $a+b=\max \{a, b\}$ and $a \cdot b=\min \{a, b\}$ then $(R ;+$,$) \quad is an h$-hemiregular hemiring.

Definition 3.12: The hemiring $R$ is h-intra hemiregular iff for each $x \in R$ there exists $a_{i}, a_{i}^{\prime}, b_{j}, b_{j}^{\prime}, z \in R$ such that

$$
x+\sum_{i=1}^{m} a_{i} x^{2} a_{i}^{\prime}+z=\sum_{j=1}^{n} b_{j} x^{2} b_{j}^{\prime}+z
$$

Example $3.12(a)$ : Let $R=\{0, a, b\}$ be a set with an addition operation + and multiplication operation $\cdot$ as follows:

\begin{tabular}{c|ccc}
+ & 0 & $\mathrm{a}$ & $\mathrm{b}$ \\
\hline 0 & 0 & $\mathrm{a}$ & $\mathrm{b}$ \\
$\mathrm{a}$ & $\mathrm{a}$ & $\mathrm{a}$ & $\mathrm{b}$ \\
$\mathrm{b}$ & $\mathrm{b}$ & $\mathrm{b}$ & $\mathrm{b}$
\end{tabular}

\begin{tabular}{c|ccc}
. & 0 & $\mathrm{a}$ & $\mathrm{b}$ \\
\hline 0 & 0 & 0 & 0 \\
$\mathrm{a}$ & 0 & $\mathrm{a}$ & 0 \\
$\mathrm{~b}$ & 0 & 0 & $\mathrm{a}$
\end{tabular}

Then $R$ is an $h$-intra hemiregular hemiring

\section{Structure of Simple Hemirings}

Definition 4.1: An element $a$ of a hemiring $R$ is infinity if $a+r=a ; \forall r \in R$.

Example 4.1 (a): In the hemiring $(R=\{0, a\} ;+, \cdot)$ the element $a$ is an infinity element of $R$.

Definition 4.2: A hemiring $R$ with unity is simple if 1 is infinity, that is to say

$a+1=1 ; \forall a \in R$.

Example 4.2 (a): The hemiring $(R=\{0,1\} ;+, \cdot)$ is a simple hemiring, where + and $\cdot$ are defined by 


\begin{tabular}{l|ll}
+ & 0 & 1 \\
\hline 0 & 0 & 1 \\
1 & 1 & 1
\end{tabular}

\begin{tabular}{l|ll}
$\cdot$ & 0 & 1 \\
\hline 0 & 0 & 0 \\
1 & 0 & 1
\end{tabular}

Theorem 4.3: A simple hemiring is an additive idempotent hemiring.

Proof: Let $(R ;+, \cdot)$ be a simple hemiring.

Since $(R ;+, \cdot)$ is simple, for any $\forall a \in R$, we have

$a+1=1$

Now, $a=a .1=a(1+1)=a+a$

$\because a=a+a$

Therefore, $(R ;+, \cdot)$ is an additive idempotent hemiring.

Proposition 4.4: The following conditions on a hemiring $R$ are equivalent:

(1) $R$ is simple;

(2) $a=a b+a ; \forall a, b \in R$;

(3) $a=b a+a ; \forall a, b \in R$.

Proof: (1) $\Leftrightarrow$ (2).

Assume (1), if $a, b \in R$, then,

$a=a .1=a(1+b)=a+a b$, proving (2).

Conversely, if (2) holds, then

$1+b=1+1 b=1 ; \forall b \in R$, proving (1).

(1) $\Leftrightarrow(3)$.

Assume (1), if $a, b \in R$, then,

$a=1 \cdot a=(b+1) a=b a+a$, proving (3).

Conversely, if (3) holds, then

$b+1=b \cdot 1+1=1 ; \forall b \in R$, proving (1).

Theorem 4.5: For a hemiring $R$ the following conditions are equivalent:

(1) $R$ is simple and multiplicatively idempotent;

(2) $(a+b)(a+c)=a+b c ; \forall a, b, c \in R$;

(3) $a, b \in R$, then $a+b=a \Leftrightarrow a b=b=b a$.

Proof: $(1) \Leftrightarrow(2)$.

Assume (1) by proposition 4.4 we have,

$$
\begin{aligned}
(a+b)(a+c) & =a^{2}+b a+a c+b c \\
& =a+b a+a c+b c \\
& =a+a c+b c \\
& =a+b c
\end{aligned}
$$

Thus, we have (2).

Conversely, assume (2).

If $a \in R$ then by (2)

$a^{2}=(a+0)(a+0)=a+0.0=a$

$I^{+}(R)=R$.

If $a, b \in R$, then,

$a b+a=(a+0)(b+1)=a+0 \cdot 1=a$ and so, by proposition $4.4, R$ is simple.

(1) $\Leftrightarrow(3)$.

Assume (1), and let $a$ and $b$ are element of $R$.

If $a+b=a$ then, by (2) we have

$a b=(b+a)(b+0)=b+a \cdot 0=b$

Similarly, $b a=(b+0)(b+a)=b+0 \cdot a=b$

Conversely, if $a b=b$ then by proposition 4.4,

$a+b=a+a b=a$.

Now assume (3).

If $b \in R$ then,

$1 \cdot b=b$

So,

$1+b=1$.

Therefore $R$ is simple.

In particular, it is additively idempotent.

Hence for each $a \in R$ we have $a+a=a$ and so

$a^{2}=a$.

Thus is multiplicatively idempotent as well.

Theorem 4.6: For each element $a$ of a simple hemiring, let $S(a)=\{0\} \cup\{r \in R: r+a=1\}$. Then

$S(a)=\{0\} \cup\{r \in R: r+a=1\}$ isa

sub-hemiring of $R$ for each $a \in R$;

$S(a) \cap S(b)=S(a b) ; \forall a, b \in R$.

Proof: Since $R$ is simple, we clearly have $1 \in S(a)$.

Therefore we must show that if $r, r^{\prime} \in S(a)$, then $r+r^{\prime}$

and $r r^{\prime}$ belong to $S(a)$. This is immediate if one of $r, r^{\prime}$ is 0 and so we can assume that both are non-zero.

In that case,

$r+a=1=r^{\prime}+a$

And so

$$
\begin{aligned}
& r+r^{\prime}+a=\left(r+r^{\prime}\right)+a \cdot 1 \\
& =\left(r+r^{\prime}\right)+a \cdot(1+1) \\
& =\left(r+r^{\prime}\right)+a+a \\
& =(r+a)+\left(r^{\prime}+a\right) \\
& =1+1 \\
& =1
\end{aligned}
$$

Establishing that $r+r^{\prime} \in S(a)$.

Moreover,

$1=1+a$

$=1 \cdot 1+a$

$=(r+a) \cdot\left(r^{\prime}+a\right)+a$

$=r r^{\prime}+r a+a r^{\prime}+a^{2}+a$

$=r r^{\prime}+(r a+a)+a r^{\prime}+a^{2}$

$=r r^{\prime}+a+a r^{\prime}+a^{2}$

[By proposition 4.4]

$=r r^{\prime}+\left(a+a r^{\prime}\right)+a^{2}$

$=r r^{\prime}+a+a^{2}$

[By propostion 4.4]

$=r r^{\prime}+\left(a+a^{2}\right)$

$=r r^{\prime}+a$

[By propostion 4.4]

Proving that $r r^{\prime} \in S(a)$.

Thus $S(a)$ is a sub-hemiring of $R$.

(2). If $0 \neq r \in S(a b)$, then $r+a b=1$.

So by proposition 4.4 ,

$$
\begin{aligned}
1 & =1+a \\
& =r+a b+a \\
& =r+a
\end{aligned}
$$


Proving that $r \in S(a)$.

Similarly $r \in S(b)$ and so,

$r \in S(a) \cap S(b)$.

Conversely suppose, $0 \neq r \in S(a) \cap S(b)$,

then,

$$
\begin{aligned}
1 & =1+r \\
& =(r+a)(r+b)+r \\
& =r^{2}+a r+r b+a b+r \\
& =a b+r
\end{aligned}
$$

And so $r \in S(a b)$.

Thus $S(a) \cap S(b)=S(a b)$.

\section{Conclusion}

In this paper we characterize some classes of hemirings especially zerosumfree hemiring, idempotent hemiring and regular hemiring. Finally we also describe simple hemiring. These structural properties will be helpful to the readers as well as researchers to formulate and characterize some diverse areas of mathematics such as automata theory, formal language theory, graph theory, combinatorial theory and so on.

\section{References}

[1] J. S. Golan, "Semirings and Their Applications", Kluwer Acad. Publ. (1999).

[2] J. S. Golan, "Semirings and Affine Equations over Them: Theory and Applications" Kluwer Academic Publishers (1999).

[3] U. Hebisch and H. J. Weinert, "Semirings: Algebraic Theory and Applications in theComputer Science", World Scientific, 1998.

[4] Jianming Zhan, Wiesław A. Dudek, "Fuzzy h-ideals of hemirings", Information Sciences 177 (2007), pp. 876-886.

[5] J. N. Mordeson and D. S. Malik, "Fuzzy Automata and Languages, Theory and Applications", Computational Mathematics Series, Chapman and Hall/CRC, Boca Raton 2002.

[6] Muhammad Shabir, Nosheen Malik, Tahir Mahmood,
"Characterizations of hemirings by The properties of their interval valued fuzzy Ideals", Annals of Fuzzy Mathematics and Informatics 3 (2), (April 2012), pp. 229-242.

[7] M. Gulistan, M. Shahzad, S. Ahmed and M. Ilyas, "Characterization of Gamma Hemirings By Generalized Fuzzy Gamma Ideals", Application and Applied Mathematics, Vol. 10. Issue 1 (June 2015), pp. 495-520.

[8] Huajun $\mathrm{Wu}$ and Jianming Zhan, "Soft Hemiring Related to Fuzzy Set Theory” KYUNGPOOK Math. J. 52 (2012), pp. 61-79.

[9] K. Ray Chowdhury, Md. Yasin Ali, A. Sultana, N. K. Mitra and A. F. M. Khodadad Khan, "On Matrices Over Path Algebra", Annals of Pure and Applied Mathematics 11 (2), 2016, pp. 45-55.

[10] Y. Q. Yin and H. X. Li, "The Characterizations of h-hemiregular hemirings and h-intra hemirings", Inform. Sci. 178 (2008) pp. 3451-3464.

[11] Huajun Wu, Jianming Zhan, "The characterizations of some kinds of soft hemirings", Annals of Fuzzy Mathematics and Informatics 3 (2), (April 2012), pp. 267- 280.

[12] K. Ray Chowdhury, A. Sultana, N. K. Mitraand A. F. M. Khodadad Khan, "Some Structural Properties of Semirings", Annals of Pure and Applied Mathematics, 5 (2) (2014) pp. 158-167.

[13] W. A. Dudek, M. Shabir and M. Irfan Ali, “ $(\alpha, \beta)$ fuzzy ideals of hemirings", Comput. Math. Appl. 58 (2009) pp. 310 321.

[14] Muhammad Shabir and Tahir Mahmood,"Hemirings characterized by the properties of their fuzzy ideals with thresholds", Quasigroups and Related Systems 18 (2010), pp. 195-212.

[15] D. R. La Torre, "On $h$-ideals and $k$-ideals in Hemirings", Publ. Math. Debrecen, 12 (2), pp. 219-226, 1965.

[16] W. A. Dudek, "Special types of intuitionistic fuzzy left $h$-ideals of hemirings", Soft Comput., 12 (4), pp. 359-364, 2008.

[17] M. Zhou and S. li, "Application of bipolar fuzzy theory to hemiring", International Journal of Innovative Computing, Information and Control Volume 10 (2), April, 2014, pp 767-781.

[18] N. Sulochana and T. Vasanthi, "Structure of Some Idempotent Semirings", Journal of Computer and Mathematical Science, Vol, 7 (5), pp. 294-301, 2016. 